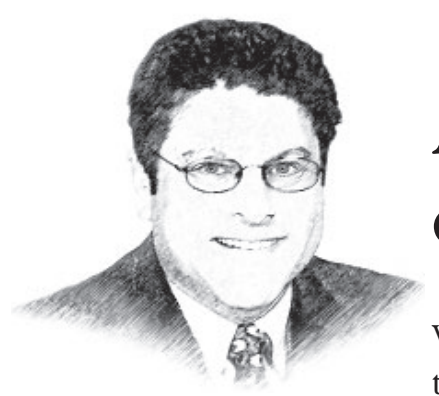

\title{
A few pearls can help prepare the mind
}

While the amount of knowledge about complex diseases is expanding, the amount of time we can spend face to face with an individual patient - and to read up on his or her problems after the encounter-is shrinking. And while medical care is depending more on subspecialists, it seems to be getting harder to get a patient in to see one. Thus, general internists and specialists increasingly need to look at the whole patient to initiate care accurately and to triage consults efficiently.

We need to recognize the diverse problems that patients with potential multisystem disease can develop, lobby when necessary for them to be seen promptly by the relevant specialists, and initiate appropriate diagnostic testing and management in less-urgent scenarios. Most of us need frequent refreshers on the clinical manifestations of these disorders so that we can recognize them when they appear unannounced in our exam rooms.

The caregiver with a prepared mind is more likely to experience the diagnostic epiphany, and then use point-of-care references to hone in on the details. With many patients and clinical conundrums, the basics matter.

Dr. Chester Oddis, in this issue of the Journal (page 656), reviews the basics of several primary muscle disorders. He discusses, in a case-based format extracted from his recent Medicine Grand Rounds presentation at Cleveland Clinic, nuances of specific diagnoses and the clinical progression of diseases that are critical to be aware of in order to recognize and manage them, and expeditiously refer the patient to our appropriate subspecialty colleagues.

Major challenges exist in recognizing the inflammatory myopathies and their mimics early in their course. These are serious but uncommon entities, and in part because patients and physicians often attribute their early symptoms to more-common causes, diagnosis can be elusive- until the possibility is considered. We hope that Dr. Oddis's article will make it easier to rapidly recognize these muscle disorders.

Patients often struggle to explain their symptoms of early muscle dysfunction. Since patients often verbalize their fatigue as "feeling weak," we often misconstrue complaints of true muscle weakness (like difficulty walking up steps) as being due to fatigue. Add in some anemia from chronic inflammation and some "liver test" abnormalities, and it is easy to see how the recognition of true muscle weakness can be delayed.

We can tease muscle weakness from fatigue or dyspnea by asking the patient to specifically and functionally describe their "weakness," and then by asking pointed questions: "Do you have difficulty getting up from the toilet without using your arms? Do you have trouble brushing your hair or teeth?" Physical examination can clearly help here, but without routine examination of muscle strength in normal fragile elderly patients, the degree of muscle weakness can be difficult to assess. Likewise challenging is detecting the early onset of weakness by examination in a $280-1 b$ power-lifter. 
Obtaining an accurate functional and behavioral history is often critical to the early recognition of muscle disease. Muscle pain, as Dr. Oddis notes, is not a characteristic feature of many myopathies, whereas, paradoxically, the coexistence of new-onset symmetrical small-joint pain (especially with arthritis) along with muscle weakness can be a powerful clue to the diagnosis of an inflammatory myopathy.

An elevated creatine kinase $(\mathrm{CK})$ level generally points directly to a muscle disease, although some neurologic disorders are associated with elevations in $\mathrm{CK}$, and the entity of benign "hyperCKemia" must be recognized and not overmanaged. The latter becomes a problem when laboratory tests are allowed to drive the diagnostic evaluation in a vacuum of clinical details.

A more common scenario is the misinterpretation of common laboratory test abnormalities in the setting of a patient with "fatigue" or generalized weakness who has elevations in aspartate aminotransferase (AST) and alanine aminotransferase (ALT). Although AST and ALT are often called "liver function tests," these enzymes are also abundant in skeletal muscle, and since they are included on routine biochemical panels, their elevation often leads to liver imaging and sometimes even biopsy before anyone recognizes muscle disease as the cause of the patient's symptoms and laboratory test abnormalities. Hence, a muscle source (or hemolysis) should at least be considered when AST and ALT are elevated in the absence of elevated alkaline phosphatase or gamma-glutamyl transferase.

When evaluating innumerable clinical scenarios, experienced clinicians can most certainly generate similar principles of diagnostic reasoning, based on having a few fundamental facts at their fingertips. Increasing the chances of having a prepared mind when confronted with a patient with a less-than-straightforward set of symptoms is one of my major arguments in support of continuing to read and generate internal medicine teaching literature and to attend and participate in clinical teaching conferences such as Medicine Grand Rounds. It is also why we will continue to appreciate and publish presentations like this one in the Journal.

I don't expect to retain all the details from these and similar papers, and I know we all carry virtually infinite databases in our pockets. But keeping a few clinical pearls outside of my specialty in my head comes in handy. Having a prepared mind makes it much easier to converse with patients, to promptly initiate appropriate testing, plans, and consultations, and to then decide what to search for on my smartphone between patients.

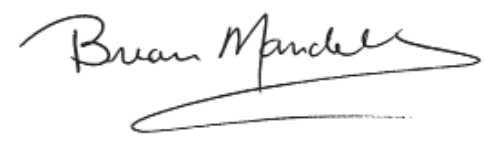

BRIAN F. MANDELL, MD, PhD Editor in Chief 